\title{
Tracking Control for High-Performance Motion of a Hydraulic Infinite Linear Actuator
}

Martin Hochwallner, Lie Pinto and Petter Krus

The self-archived postprint version of this journal article is available at Linköping University Institutional Repository (DiVA):

http://urn.kb.se/resolve?urn=urn:nbn:se:liu:diva-153684

N.B.: When citing this work, cite the original publication.

Hochwallner, M., Pinto, L., Krus, P., (2018), Tracking Control for High-Performance Motion of a Hydraulic Infinite Linear Actuator, IEEE/ASME transactions on mechatronics, 23(6), 2782-2793. https://doi.org/10.1109/TMECH.2018.2871865

Original publication available at:

https://doi.org/10.1109/TMECH.2018.2871865

Copyright: Institute of Electrical and Electronics Engineers (IEEE)

http://www.ieee.org/index.html

(C)2018 IEEE. Personal use of this material is permitted. However, permission to reprint/republish this material for advertising or promotional purposes or for creating new collective works for resale or redistribution to servers or lists, or to reuse any copyrighted component of this work in other works must be obtained from the IEEE. 


\title{
Tracking Control for High Performance Motion of a Hydraulic Infinite Linear Actuator
}

\author{
Martin Hochwallner, Lie Pablo Grala Pinto, and Petter Krus
}

\begin{abstract}
The concept of the Hydraulic Infinite Linear Actuator, HILA, consists of two double-acting cylinders with hydraulically detachable pistons driving a common rod. Alternatingly one cylinder engages and drives the load while the other retracts; the actuator thus works in a kind of rope-climbing motion.

The actuator has three degrees of freedom, DoFs, which are temporarily coupled compared with one in a conventional cylinder. Hence, the actuator is a hybrid system with both, continuous and discrete states and control inputs.

This contribution presents a tracking control problem and a solution for high-performance motion, which is interpreted as utilizing the hardware limits in the sense of velocity, accuracy, smoothness and usability. The concept is demonstrated by simulation for the case of a rest-to-rest motion, but the intended use-cases also include arbitrary trajectories.
\end{abstract}

Index Terms-linear hydraulic actuator, infinite motion, tracking problem, hybrid system, hydraulic servo system

\section{INTRODUCTION}

In hydraulics, linear motion is actuated directly by hydraulic cylinders or indirectly by hydraulic rotational motors connected to rack and pinion or leading screw type mechanisms. Motors, in contrast to cylinders, provide infinite stroke by the concept, but need mechanisms to convert rotational into translational motion. The presented actuator realizes infinite stroke for direct linear actuation, see [1], [2], [3].

Conventional cylinders have mechanical issues, buckling, and dynamical issues, low natural frequency, when long stroke is required. Additionally, the behavior depends strongly on the piston position. A conventional cylinder system has to be designed and manufactured for a specific stroke length which can later not be adjusted. The goal of the development effort, where this article contributes to, is to develop an alternative hydraulic linear actuator without these issues. The intention is not to design an actuator for one defined application but in a technology-push manner [4] investigate the opportunities and weaknesses of the actuator concept. Further on, it is a stated goal to develop an agile component [5] which is as independent of the actual application as possible.

Conventional Hydraulic Linear Actuators, i.e. valve controlled cylinders, are common and mature components of hydraulic systems. Ongoing research focuses on secondary control with multi-chamber cylinders [6], various concepts of digital hydraulics [7], individual metering [8], and advanced control concepts [9], [10]. A further topic is sensor-less positioning with stepper drives [11]. Hydraulic stepper drives

Martin Hochwallner, Lie Pablo Grala Pinto and Petter Krus are with the Division of Fluid and Mechatronic Systems, Linköping University, SE-581 83 Linköping, Sweden, e-mail: martin.hochwaller@liu.se.

Manuscript received 2017-05-11; . Date of publication 2018-09-24.

DOI: 10.1109/TMECH.2018.2871865 like those in [12], [13], [14] are similar to the presented concept. The conceptual difference is that in stepper drives, the pistons always move from end-stop to end-stop using the number of strokes, steps, as the unit of motion. Intermediate positions and continuous motion are not part of the hydraulic stepper drive concept.

The commercially available systems most similar to the presented actuator are strand jacks, e.g. from ENERPAC [15]. Strand jacks are lifting devices pulling bundles of steel cables in a linear motion and are used in bridge construction. The velocity reached is small and thus measured in meters per hour. The presented Hydraulic Infinite Linear Actuator in contrast is intended to be a system comparable with conventional cylinders. The actuator realizes dynamic motion, whereby the velocity is measured in meter per second. The simulated actuator reaches $0.25 \mathrm{~m} / \mathrm{sec}$ driving a $20 \mathrm{kN} / 2000 \mathrm{~kg}$ load.

The working principle of the actuator, see Figure 1, is that cylinder A engages, i.e. clamps the rod, and drives the load while the other cylinder, B, disengages and retracts. Before cylinder A reaches the end of its stroke, cylinder B engages and cylinder $\mathrm{A}$ disengages. The motion continues by means of the two cylinders alternating their roles of driving the load and retracting. Engaging is realized by hydraulically controllable clamping elements.

This contribution presents a trajectory tracking solution for HILA with two cylinders. The goal is to encapsulate the hybrid system so that the application does not have to deal with its complexity. The main contribution is the inverse kinematics, which translates the actuator trajectory into cylinder trajectories and governs the engaging/disengaging and the load shifting between the cylinders. The problem solved is actuator motion of the kind "from rest to rest", but the concept is not limited to this. The topic of generating more general trajectories has been solved by other researches, for example [16], [17], [18] and is not handled here.

With a test rig, provided by industry, the working principle has been validated, but the rig is inadequate for studying fast, accurate motion. The test rig realizes a different system presented in [2]. Validating the high-performance motion addressed in this contribution requires a distinctly more advanced test rig.

\section{CONCEPT}

The actuator can be described as a system involving continuous dynamics and discrete states and it can thus be categorized as a hybrid system like investigated in [19]. The continuous states of the actuator are formed by the hydraulic cylinders, and the rod / load. There is one mechanical DoF per cylinder, 


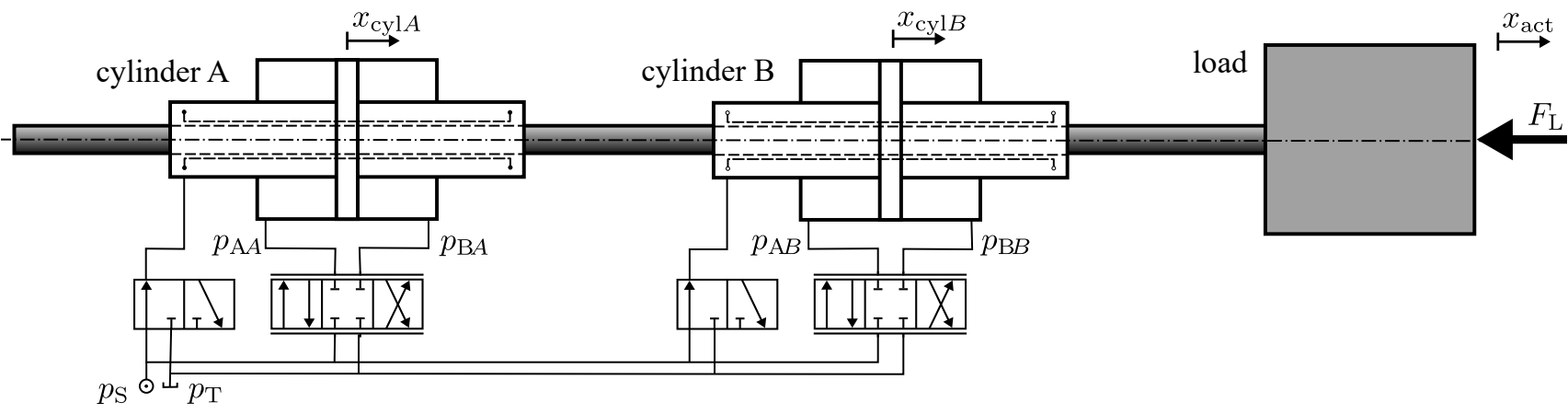

Fig. 1. System: Two symmetric double-acting cylinders temporarily hydraulically detachable from the common rod driving the load. Sensors: position of the cylinders, $x_{\mathrm{cyl} A}, x_{\mathrm{cyl} B}$ and the load or actuator, $x_{\mathrm{act}}$, pressure in each cylinder chamber, $p_{\mathrm{A} A}, p_{\mathrm{B} A}, p_{\mathrm{A} B}, p_{\mathrm{B} B}$, as well as supply, $p_{\mathrm{S}}$, and tank line, $p_{\mathrm{T}}$. The position sensor for the load may be omitted. The control inputs are the valves' input signals.

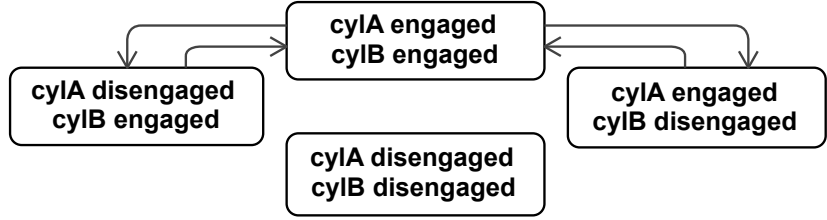

Fig. 2. Discrete states of the hybrid system and the transitions of the gait investigated in this contribution.

$x_{\mathrm{cyl} A}$ and $x_{\mathrm{cyl} B}$ in Figure 1, and one DoF of the load, $x_{\text {act }}$, i.e. there are in total three mechanical DoFs in this system. The discrete states are formed by the clamping elements of the two cylinders which can be engaged or disengaged and thus 4 states exist, see Figure 2. Depending on these discrete states, the DoF of each cylinder can be coupled with the DoF of the load. Thus, the DoF of cylinder A or the DoF of cylinder B or the DoFs of both are temporarily eliminated.

The patterns in which the actuator switches between these states form the gaits in which the actuator can be operated. This contribution focuses on the gait where alternately one cylinder disengages to retract, i.e. there is always at least one cylinder engaged to drive the load. Other gaits to investigate in future are for example the gait where both cylinders are engaged and join their forces. Also of interest is the gait where a cylinder accelerates the load, then both cylinders disengage and the load moves further in a ballistic way [3].

\section{A. Control Concept}

The goal of the control concept in this work is to move a load along a trajectory from A to B. The problem is known as the tracking problem. Further on, the control concept will exploit the limitations of the system without violating them. The same is valid for limitations imposed by the application. System limitations here are for example velocity limitations due to limitations of the cylinder and the capabilities of the hydraulic power supply, and limitations in acceleration and jerk due to the limitations in the valve dynamics.

Therefore, a control scheme with two degrees of freedom [22], [23] is applied, which combines feedback to control the tracking error with feedforward. The feedforward component calculates the control signal for ideal conditions based on the desired trajectory. The desired trajectory is generated so that the system stays within the system and the application limits. Hence, there are two major topics to address: the feedback controller and the trajectory generation.

Figure 3 shows the control scheme for an actuator with two cylinders. For each cylinder, the inner feedback loops are closed by the cylinder controllers. Depending on the discrete states, the system dynamics change. Hence, the controller parameters are switched via scheduler in the sense of gain scheduling [24]. The feedforward component itself is made up of the actuator trajectory generator, the inverse kinematics, and the cylinder controllers. The cylinder controllers calculate the control signals needed by the proportional valves based on the desired cylinder trajectories. Additionally, the cylinder controllers trigger the on/off valves and thus control the engagement of the cylinders based on the signals from the inverse kinematics.

The actuator trajectory generator plans the trajectory of the actuator based on the input from the application and defined limits. The limitations are formulated as upper limits on velocity, acceleration, jerk, and higher order derivatives, if needed. The internal state, e.g. the position of the cylinders and the engagement, are explicitly not considered. The presented actuator trajectory generator realizes motion of the kind "from rest to rest". More general cases can be solved by substituting the block actuator trajectory generator in Figure 3, with trajectory generators from e.g. [16], [17]. The concept and the control schematic thereby do not change. An alternative way to utilize the control concept is to use the interface, marked alternative interface in Figure 3 directly by the application. This is for example the case with humans in the loop.

The inverse kinematics translates the momentarily actuator trajectory into the trajectories of the cylinders. It thereby has to determine which cylinder is to drive the load and thus be engaged. During the changeover between the cylinders driving the load, the load has to be actively shifted between them to avoid extensive jerk being introduced into the system, see [1]. This is realized by controlling the force each cylinder shall drive so that when disengaging the cylinder will not drive the load. Therefore, the generated signals contain information about the expected load distribution between the cylinders.

The controller is intended to exploit the limitations of the 


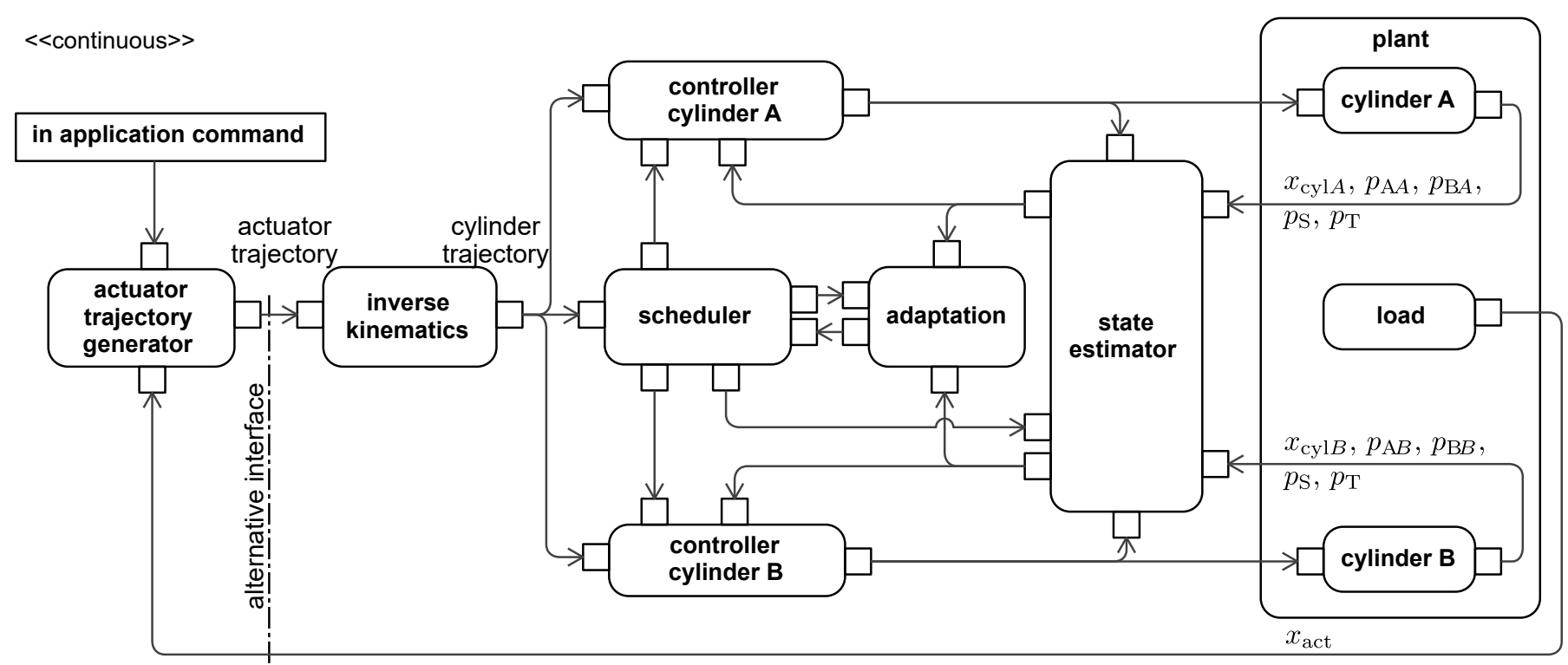

Fig. 3. Control scheme for two cylinders. SysML activity diagram [20], [21]

system without violating them. The system limitations can be seen as a budget for the whole controller. Parts of this budget can thus be allocated to the blocks in the control scheme. To reach an optimal configuration, these budgets can be balanced between the blocks, cylinder controllers, inverse kinematics, and actuator trajectory generator. The expected disturbances on the system require a part of the budget for the feedback control. The inverse kinematics require a part of the budget for retraction and load shift. The remaining budget is available through the actuator trajectory generator for the application.

\section{The System Model}

The system consists of common hydraulic actuators, the engaging and disengaging mechanisms and a load. Various load cases are discussed in [1]. Often, a load can be simplified to a mass with additional force. Therefore, the investigated application is lifting a mass, $2000 \mathrm{~kg}$ and $20 \mathrm{kN}$, as this is a simple but generic load case. The derivation of equations for common hydraulic actuators, see Figure 1, can be found in various textbooks. This work is based on the equations derived in [10]. The engaging and disengaging subsystem is the key subsystem in HILA, the enabler of the concept and is not standard and requires deeper analyses. Its purpose is to lock and unlock the cylinders to the common rod.

\section{A. The Engaging and Disengaging Subsystem}

The proposed control concept assumes only a few characteristics of the engaging / disengaging subsystem as: a) When engaged, the transferable force is beyond a specified lower limit; b) When disengaged, the disturbance force is below a specified upper limit; c) The engaging process takes less than a specified upper limit duration $T_{\text {engage }}$; d) The disengaging process takes less than a specified upper limit duration $T_{\text {disengage. }} T_{\text {engage }}$ and $T_{\text {disengage }}$ consider all delays from the trigger in the software, until the required force is actually transferable. These assumptions ensure that the system is robust against variations, for example caused by the influence of temperature onto the dynamics.

1) The Clamping Element: In the considered system, the engaging / disengaging is realized with the commercially available off-the-shelf hydraulically actuated hub-shaft connection Octopus from ETP [25], see Figure 4. The hub-shaft connection transfers the cylinder force due to friction of the hydraulically actuated membrane being pressed against the rod. The friction is modeled as Coulomb friction as follows, see also [2].

$$
F_{\mathrm{c}}= \begin{cases}F_{\mathrm{c} 0} & \text { when: } p_{\mathrm{c}}<p_{\mathrm{c} 0} \\ F_{\mathrm{c} 0}+\mu A_{\mathrm{c}}\left(p_{\mathrm{c}}-p_{\mathrm{c} 0}\right) & \text { else }\end{cases}
$$

$F_{\mathrm{c} 0}$ is the remaining friction force caused for example by scrappers. $p_{\mathrm{c}}$ is the oil pressure in the connection and $p_{\mathrm{c} 0}$ is the minimum pressure where the connection starts to engage. $\mu$ is the friction parameter and $A_{\mathrm{c}}$ the effective area where $p_{\mathrm{c}}$ acts to create the normal force to create the friction force.

2) The Actuation System: The clamping element is actuated by pressure $p_{\mathrm{c}}$ in the inner volume of the hub-shaft connection.

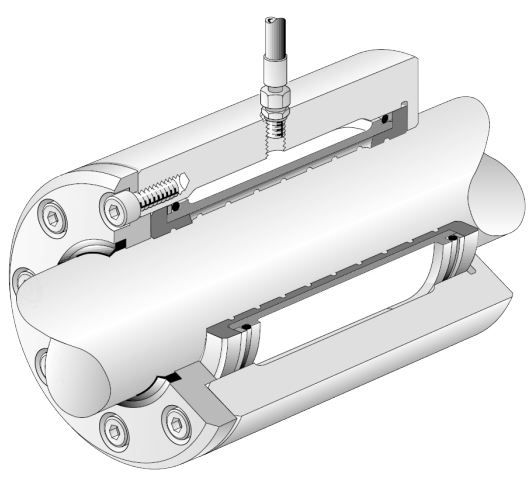

Fig. 4. Hydraulic hub-shaft connection [25] 


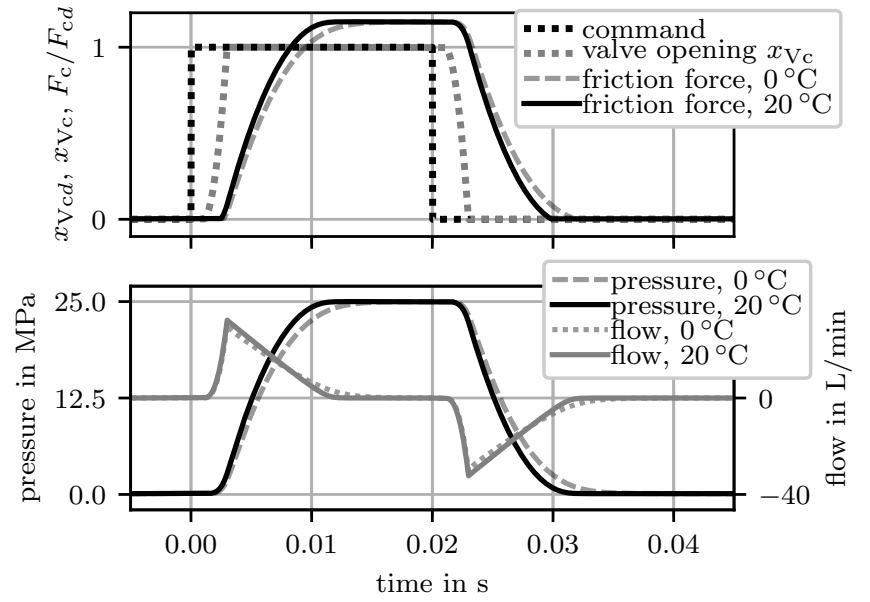

Fig. 5. Engaging / Disengaging Subsystem: Simulation results of an engaging and a disengaging process. The transferable force $F_{\mathrm{c}}$ exceeds the design force $F_{\mathrm{c} d}$ by the factor 1.15 .

A fast on/off valve connected to the clamping element via a hose controls the pressurization and depressurization.

The dynamics of the pressurization / depressurization are mainly dependent on the valve dynamics and the pressure build-up and release in the volume. Hence, accepting the limited fidelity, the actuation system is modeled as volume pressurized through a resistance and a valve. It is assumed that the pressure and tank connections of the valve are supported by accumulators. The flow resistance in the hose is modeled temperature dependent according to Hagen-Poiseuille [10].

a) Valve: The fast switching valve FSVi 4.1 [26] from Linz Center of Mechatronics $\mathrm{GmbH}$ is used. This valve features $5 \mathrm{~L} / \mathrm{min}$ at 5 bar pressure drop and a nominal switching time of $3 \mathrm{~ms}$. A summary of the "State Of The Art In Digital Valve Technology" can be found in [27]. The opening dynamics of the FSVi is approximated by a delay of $1 \mathrm{~ms}$ and constant acceleration of the poppet which opens and closes the valve in $2 \mathrm{~ms}$. This simple model fits sufficiently to the plots in the data sheet [26].

3) Results: Figure 5 show simulation results of an engaging and a disengaging process. The figure shows that the valve opens and closes in $3 \mathrm{~ms}$. Within the mentioned assumptions, the pressurization / depressurization occurs in $10 \mathrm{~ms}$ at room temperature. At $0{ }^{\circ} \mathrm{C}$, the pressurization still occurs in $10 \mathrm{~ms}$, but in the case of depressurization the friction force is at approximately $1.5 \mathrm{kN}$ at $10 \mathrm{~ms}$ and reaches minimum approximately $2 \mathrm{~ms}$ later. Thus, in the case of $0{ }^{\circ} \mathrm{C}$ the assumed characteristics do not hold. Assuming the friction parameters specified in the data sheet [25], the transferable force reaches approximately $23 \mathrm{kN}$ and exceeds the actuator design force by the factor 1.15 .

4) Slip: In this contribution, everything resulting in deviation between the actuator position $x_{\text {act }}$ and the accumulated position of the cylinders is named "slip". The accumulated position is the theoretical actuator position caused by the cylinders if ideal clamping occurs. Slip also includes the effects of position deviations of the cylinders while engaging/disengaging. Most effects cause slip during the engaging and disengaging phase but not during the driving phase. Others, like overload, do not occur in well-dimensioned systems.

\section{TRAJECTORY}

The proposed concept divides the task of trajectory planning into two tasks. Fist, the actuator trajectory generator, which covers the application side and second, the cylinder trajectory planing, called inverse kinematics, which translates the desired actuator trajectory into the desired cylinder trajectory, see Figure 3. The actuator trajectory generator generates the trajectory of the actuator or load based on application inputs. It does not consider details of the cylinder configuration, such as the cylinder stroke length or the cylinders' positions, but only general limitations imposed by the actuator. The inverse kinematics translate the actuator trajectory in trajectories for each cylinder based on the currently desired state of the cylinder. Repeating an actuator trajectory will usually not result in repeating the cylinder trajectories as the initial situation may have changed.

\section{A. Actuator Trajectory}

The considered application input, called application command in Figure 3, has the form of 'go to position X'. Limitations from the application side are for example upper limits on acceleration or jerk. Limitations from the actuator side, like the upper limit of the valve opening, are translated into trajectory properties as there are upper limits for velocity, acceleration, and jerk. Depending on the chosen order of the trajectory which depends on the chosen cylinder controller, limits on higher order derivatives may be in place. The trajectory shall be smooth, i.e. $m$-times continuously differentiable, $C^{m}$. In the simulated example, a third order trajectory is used.

The trajectory is specified as the momentaneous values of the desired actuator position $x_{\text {act } d}(t)$ and its time derivatives $\frac{\mathrm{d}^{i}}{\mathrm{~d} t^{i}} x_{\text {act } d}(t)$ for $i=1$..n. No past or future values are required for the subsequent inverse kinematics or cylinder controllers. Trajectory generation is handled in the literature, for example in [16], [18], [17]. Here, S-curve type trajectories based on piecewise polynomials are used, see Figure 6.

1) Compensation for Slip: It is assumed that there will be a deviation between the accumulated position of the cylinders and the position of the actuator, called slip. The proposed solution is to recalculate the actuator trajectory after for example each engaging process. To realize this, a trajectory generation algorithm which can update the trajectory while the system is in motion is required. Kröger in [16] presents such an algorithm. Slip may occur while the actuator is not moving. This case can also be handled by recalculation the trajectory to the desired position. Applications may use only the accumulated position without actuator position sensor and reach sufficient accuracy as in sensor-less motion control. In the presented simulation, recalculation is not implemented.

2) Infinite Motion: The working title of the project is Hydraulic Infinite Linear Actuator. Infinite or long-distance motion control may run into numerical troubles when handling position measurements of long distances at high resolution. The proposed concept does not rely on measuring the absolute 


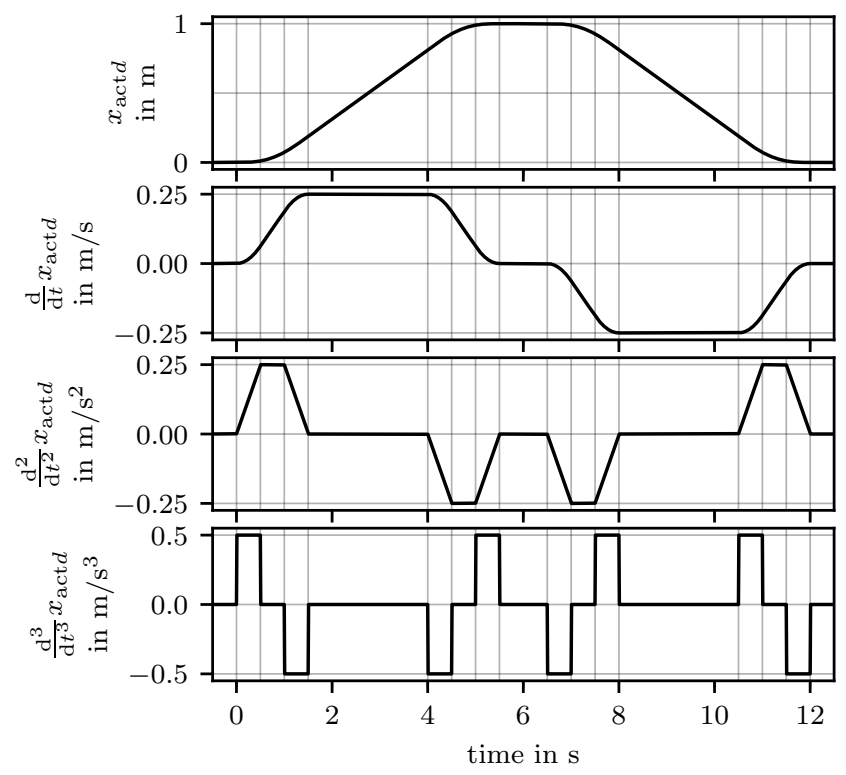

Fig. 6. Third order actuator trajectory for a motion from position $0 \mathrm{~m}$ to position $1 \mathrm{~m}$ and back. The limits for the $2^{\text {nd }}$ and $3^{\text {rd }}$ time derivation are chosen to show the sections of the trajectory and are lower than in the later simulation, Figure 12.

position but only on the direction, and in a range around the destination, on the position relative to the destination.

During the acceleration and traveling phase, the actuator trajectory generator computes the trajectory based on the direction, the knowledge that the destination is far away. When reaching the destination, in a remaining distance bigger than the deceleration phase, the position relative to the destination has to become available and the actuator trajectory can be recalculated to realize the desired stop.

\section{B. Cylinder Trajectory - Inverse Kinematics}

The generation of the cylinder trajectory has a function like inverse kinematics in robotics which translates the end-effector trajectory into the joint trajectories. In the proposed concept, it transforms the actuator trajectory into cylinder trajectories.

The inputs to inverse kinematics are the momentaneous values of the desired actuator position $x_{\text {act }}(t)$ and its time derivatives. The outputs are the momentaneous values of the cylinders' position $x_{\mathrm{cyl} j d}(t)$ and its time derivatives.

Additional to the trajectory of each cylinder, the inverse kinematics provide the underlying controller with the information about a) which cylinder is to be engaged, engagement; b) which cylinder is to drive which part of the load, load distribution; and c) what systems dynamics it has to cope with, encoded in the state, see Figure 7.

The cylinder's trajectory can be split into two sections and the transition phase between, see Figures 7 and 8 . There is the driving phase, where the cylinder is engaged and drives the load. The other cylinder is disengaged. Then there is the retracting phase, where the disengaged cylinder retracts. Between these phases are transition phases, where the load is shifted from the former to the future load-driving cylinder.

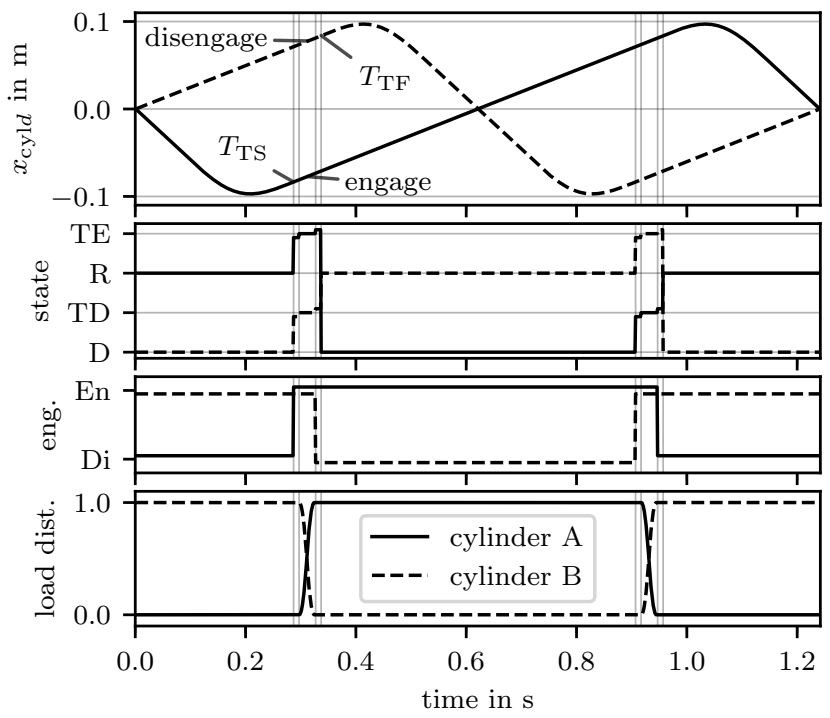

Fig. 7. One period of the cylinder trajectory. The transition phases are marked with vertical lines. The states correspond to Figure 8 and are labeled with D: driving, TD: transition disengaging, R: retracting, TE: transition engaging. The engagement is labeled with Di: disengaged and En: engaged. At $t=0 \mathrm{~s}$, cylinder B drives the load, A retracts. During the transition phase, marked with vertical lines, $T_{\mathrm{TS}}$ : transition start, $T_{\mathrm{TF}}$ : transition finish, A engages and $\mathrm{B}$ disengages. Then cylinder A drives the load while B retracts, straight, rising line and curved downward line in plot 1 respectively.

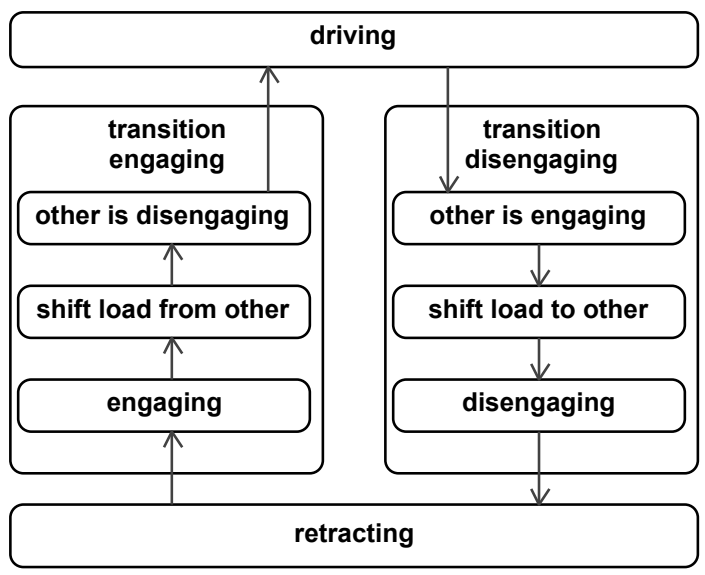

Fig. 8. State machine of one cylinder. Both cylinders follow equal state machines, but with shifted states, i.e. when one cylinder is in state driving then the other is in retracting and vice versa. SysML State Machine [20], [21].

The following requirements can be derived for the cylinder trajectories and thus the inverse kinematics.

REQ1: While not disengaged, i.e. while engaged and during the transition phases, the cylinders shall follow the given actuator trajectory.

REQ2: The retracting cylinder shall be in place for engaging and synchronized with the common rod when the condition for load shift occurs.

$R E Q 3:$ The cylinder trajectory shall stay within the limits of the system.

REQ4: The cylinder trajectory shall be smooth, i.e. $m$ times continuously differentiable, $C^{m}$.

1) Phase: Driving the load: During this phase, the cylinder is engaged with the load. Hence, the cylinder trajectory, 


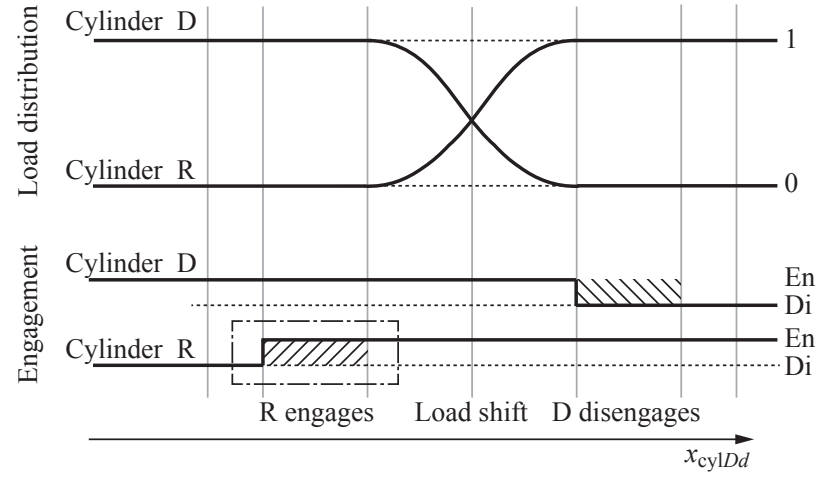

Fig. 9. Transition phase, as defined by the inverse kinematics. Principle sketch, like a detail from Figure 7 for constant velocity. The load is shifted from cylinder $\mathrm{D}$, driving, to cylinder $\mathrm{R}$, retracting. The shaded area marks the uncertainty in the engaging/disengaging process. The dashed-dotted rectangle marks the detail shown in Figure 10.

$x_{\mathrm{cyl} D d}(t)$, must be identical to the actuator trajectory, see REQ1, and is thus fully specified. The only difference is the offset in position, $x_{\text {cyl offset, } k}$, accounting for the position of the actuator, where the cylinder engaged.

$$
\begin{aligned}
x_{\mathrm{cyl} D d}(t) & =x_{\mathrm{act} d}(t)-x_{\mathrm{cyl} \text { offset, } k} \\
\frac{\mathrm{d}^{i}}{\mathrm{~d} t^{i}} x_{\mathrm{cyl} D d}(t) & =\frac{\mathrm{d}^{i}}{\mathrm{~d} t^{i}} x_{\mathrm{act} d}(t) \quad \text { for } \quad i=1 . . n
\end{aligned}
$$

2) Phase: Transition: During the transition phase, the formerly retracting cylinder engages, then the load is shifted to it and then the formerly load-driving cylinder disengages.

Two concepts of load shifting are presented and analyzed in [1]. One lets the load shift happen and thus introduces substantial jerk into the system. The other shifts the load actively between the cylinders. In this contribution, a more sophisticated active load shifting concept is proposed.

The transition phase can be split into three sub-phases, the engaging of the formerly retracting cylinder, the load shift and the disengaging of the now not load-driving cylinder. In this phase the cylinder are named after their function before entering this phase, i.e. the position is called $x_{\mathrm{cyl} D}$ even when the load has been already shifted to the other cylinder.

a) Trajectory: During the whole transition phase, there must be no relative motion between both cylinders and the common rod as this would mean relative motion under at least partial engagement, which may cause wear in the clamping element and disturbance on the load. The trajectories of both cylinders are thus governed by the actuator trajectory as in the driving phase.

b) Cylinder Engagement: The engagement and disengagement of the cylinders do not happen instantaneously. The relevant engagement time, $T_{\text {engage}}$, and disengagement time, $T_{\text {disengage }}$, are the upper limits of the duration from the trigger in the software to full engagement respectively.

In the presented concept, the formerly retracting cylinder is commanded to engage after entering the transition phase. Then, the system waits for at least $T_{\text {engage }}$ before executing the load shift. After the load shift, the formerly load-driving cylinder is commanded to disengage and after at least $T_{\text {disengage }}$

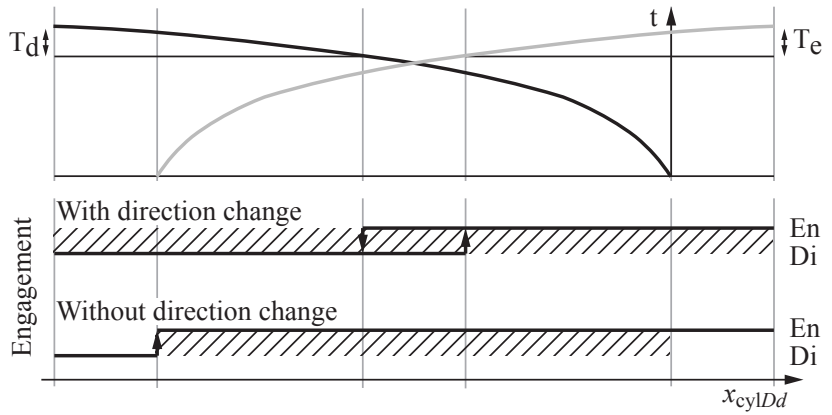

Fig. 10. Engaging phase with hysteresis for the case of direction change. This is the detail from Figure 9. The disengaging phase is equal but inverted. The lower graph shows the case without direction change as in Figure 9. The middle graph shows the case when a direction change during the engaging occurs. The upper graph illustrates the dimensioning situation, acceleration at the limit, as a time/position plot. The cylinder has to be able to engage $T_{e} \geq T_{\text {engage }}$ or disengage $T_{d} \geq T_{\text {disengage }}$ before leaving the engaging phase.

the transition phase can be left to continue with the driving and retracting phases. This process is shown in Figure 9.

In the presented concept, all these timing issues are expressed in positions of the driving cylinder, which are defined by the upper limit of the actuator velocity. Engaging / disengaging is controlled by the desired position of the driving cylinder generated by the inverse kinematics and not the actual position to eliminate the influence of position deviation and measurement noise.

This works well for the case where the actuator does not change direction while in the transition phase. Practically, such a situation could be avoided by the actuator trajectory planing considering the cylinder motions. The desire followed in this contribution is to encapsulate the inner behavior of the actuator in a way that the user / actuator trajectory planing does not have to consider the cylinder motion and transition phases. Therefore, a change in direction can not be ruled out for any cylinder position.

A solution is shown in Figure 10. A hysteresis is introduced between the switching points for the different directions. Additionally, a duration for disengaging has to be added which makes the engaging phase more than twice as long, which in many applications is not acceptable. This additional time is only relevant in the case where the direction change occurs within the engaging phase. The fact that the actuator trajectory is restricted can be used to refine this solution. Taking into account that changing direction also mean that the velocity has to cross $0 \mathrm{~m} / \mathrm{s}$ and that the acceleration has to be smaller than or equal to the defined limit leads to the velocity remaining low in the considered situation.

For the normal case, where no direction change occurs within the engaging or disengaging phase, the original concept described above is used. When a direction change is detected, the hysteresis solution with new switching points is used as long as the engaging/disengaging phase has not been left. The switching points are defined so that in every case the engaging or disengaging is finished before leaving the engaging/disengaging phase. The dimensioning case is when the direction change occurs at the border of the engaging/disengaging phase and the cylinder is accelerating at 
the acceleration limit, see Figure 10.

c) Load Distribution: When entering the transition phase, the driving cylinder drives all the external load and the retracting cylinder none. Before disengaging, the load has to be shifted from the former to the future driving cylinder, so that no jerk will be introduced into the system. Evidently both cylinders also drive their own piston mass and work against their own friction and other disturbances, which is handled in the controller. The concern in this section is the load force (static and dynamic) acting on the actuator. The load distribution between the cylinders is generated as part of the trajectory as signal $e_{R}(t)$ and $e_{D}(t)$, with a continuous range from 0.0 to 1.0 for each cylinder.

As the force on a hydraulic cylinder can not jump, the signal has to be smooth. In this contribution, a signal $C^{1}$ made-up of two polynomials of degree 2 is used. The load distribution signal is defined for both cylinders as a function of the position of the formerly load-driving cylinder, $x_{\mathrm{cyl} D}(t)$.

3) Phase: Retracting: During this phase, the cylinder is disengaged from the load and the trajectory, $x_{\mathrm{cyl} R}(t)$, called retracting trajectory, in contrast to the driving phase, is not fully specified by the given requirements.

This contribution proposes a concept where the trajectories of the two cylinders are strictly coupled. This means that the trajectory of the retracting cylinder is a function of the trajectory of the driving cylinder. This function is called $G(u)$, where $u$ is the input coordinate, i.e. the trajectory of the driving cylinder.

$$
x_{\mathrm{cyl} R}(t)=G\left(x_{\mathrm{cyl} D}(t)\right)=G(u(t))
$$

This function may be interpreted as a kind of virtual gear with a varying gear ratio coupling one coordinate to the other.

Requirement REQ4, demanding smoothness, leads to the matching conditions at the boundary to the transition phase at $T=T_{\mathrm{TS}}$ and $T=T_{\mathrm{TF}}$.

$$
\begin{aligned}
\left.x_{\mathrm{cyl} R}(t)\right|_{t=T} & =\left.x_{\mathrm{cyl} D}(t)\right|_{t=T}-x_{\mathrm{cyl} \text { offset }} \\
\left.\frac{\mathrm{d}^{i} x_{\mathrm{cyl} R}(t)}{\mathrm{d} t^{i}}\right|_{t=T} & =\left.\frac{\mathrm{d}^{i} x_{\mathrm{cyl} D}(t)}{\mathrm{d} t^{i}}\right|_{t=T} \quad \text { for } i=1 . . n
\end{aligned}
$$

Using the chain rule of differentiation it can be shown that such a function $G(u)$ has to fulfill the following conditions at position $u=x_{\mathrm{cyl} D}\left(t=T_{\mathrm{TS}}\right)$, where the retracting motion connects to the transition / driving motion and at $u=x_{\mathrm{cyl} D}\left(t=T_{\mathrm{TF}}\right)$, where the transition / driving motion connects to the retracting motion, see Figure 7.

$$
\begin{gathered}
\left.G(u)\right|_{u=x_{\mathrm{cyl} D}(t=T)}=x_{\mathrm{cyl} R}(t=T) \\
\left.\frac{\partial G(u)}{\partial u}\right|_{u=x_{\mathrm{cyl} D}(t=T)}=1 \\
\left.\frac{\partial^{i} G(u)}{\partial u^{i}}\right|_{u=x_{\mathrm{cyl} D}(t=T)}=0 \text { for } i=2 . . n
\end{gathered}
$$

for cases $T=T_{\mathrm{TS}}$ and $T=T_{\mathrm{TF}}$

In the case of a skew symmetric trajectory, the conditions for $u=x_{\mathrm{cyl} D}\left(t=T_{\mathrm{TS}}\right)$ and $u=x_{\mathrm{cyl} D}\left(t=T_{\mathrm{TF}}\right)$ are equivalent. As the system is assumed to be symmetric, the trajectory is

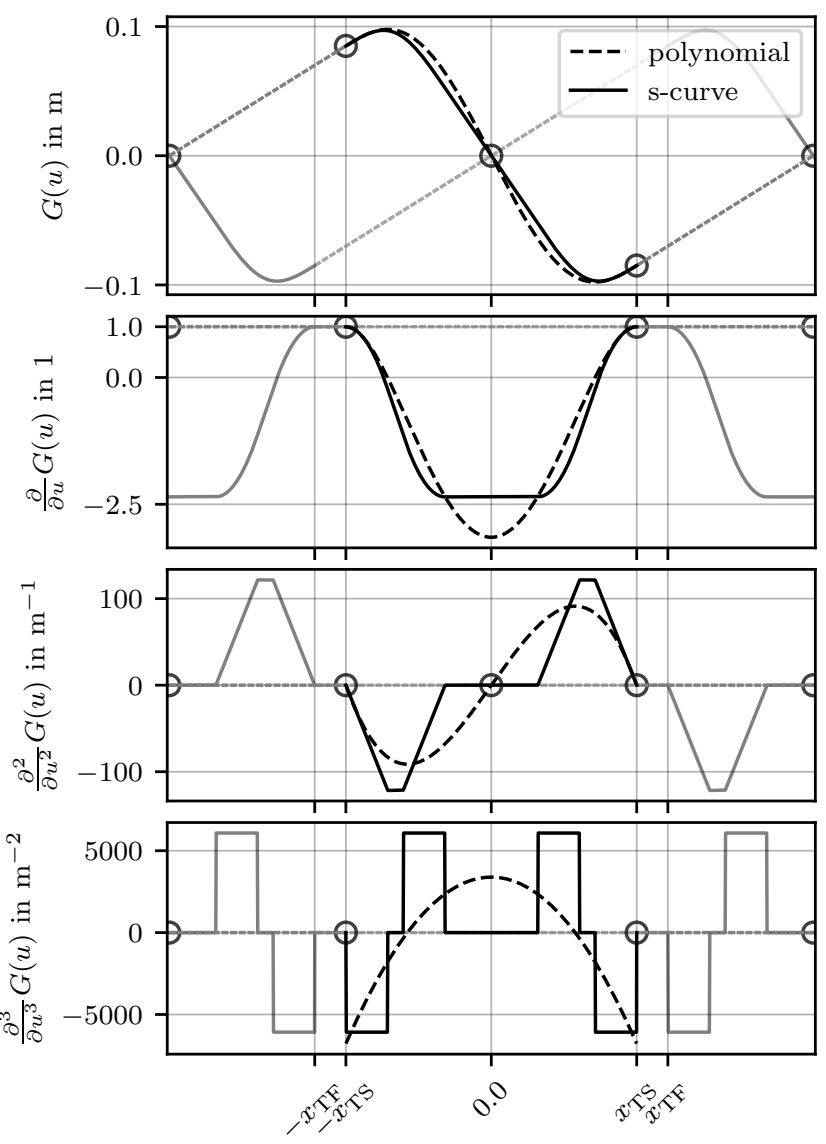

$u=x_{\mathrm{D}}$ in $\mathrm{m}$ where $x_{\mathrm{TS}}=0.070 \mathrm{~m}, x_{\mathrm{TF}}=0.0875 \mathrm{~m}$

Fig. 11. Gear function of the inverse kinematics. The graph shows two candidates for the gear function, a polynomial of degree 5 and an S-curve realized with piecewise polynomials of degree 3 . The circles mark the necessary matching conditions. The gray dashed line shows the extension of the gear function beyond the retraction phase to demonstrate the full cycle. The faded graphs show the corresponding situation for the other cylinder. $x_{\mathrm{TS}}, x_{\mathrm{TF}}$ : position of the driving cylinder where the transition phase starts and finishes.

chosen to be skew symmetric around the center position of the cylinder. All coordinates are measured from this center. This leads to the function needing to be an odd function and hence the condition for skew symmetric applies.

$$
\left.\frac{\partial^{i} G(u)}{\partial u^{i}}\right|_{u=0}=0 \text { for } i=0,2,4, . . n
$$

Candidates for such a function $G(u)$ are for example polynomials, piecewise polynomials also called S-curves or S-trajectories, B-Splines [28], or lookup-tables.

For implementation and analysis purposes the gear function can also be extended to the transition and driving phase $U_{\mathrm{DT}}$ of the trajectory and thus cover a full cycle as shown as gray dashed lines in Figure 11. Applying this function during the transition and driving phase leads to an identical function.

$$
\begin{gathered}
\left.G(u)\right|_{u \in U_{\mathrm{DT}}}=u+\text { offset } \\
\left.\frac{\partial G(u)}{\partial u}\right|_{u \in U_{\mathrm{DT}}}=1
\end{gathered}
$$




$$
\left.\frac{\partial^{i} G(u)}{\partial u^{i}}\right|_{u \in U_{\mathrm{DT}}}=0 \quad \text { for } \quad i=2 . . n
$$

The cylinder trajectory is calculated as follows by applying $G(u)$ and the chain rule of differentiation to $x_{\mathrm{cyl} D}(t)$.

$$
\begin{aligned}
x_{\mathrm{cyl} R}(t)= & G\left(x_{\mathrm{cyl} D}(t)\right) \\
\frac{\mathrm{d} x_{\mathrm{cyl} R}(t)}{\mathrm{d} t}= & \left.\frac{\partial G(u)}{\partial u}\right|_{u=\mathrm{cyl} D(t)} \frac{\mathrm{d} x_{\mathrm{cyl} D}(t)}{\mathrm{d} t} \\
\frac{\mathrm{d}^{2} x_{\mathrm{cyl} R}(t)}{\mathrm{d} t^{2}}= & \left.\frac{\partial^{2} G(u)}{\partial u^{2}}\right|_{u=\mathrm{cyl} D(t)}\left(\frac{\mathrm{d} x_{\mathrm{cyl} D}(t)}{\mathrm{d} t}\right)^{2} \\
& +\left.\frac{\partial G(u)}{\partial u}\right|_{u=x_{\mathrm{cyl} D}(t)} \frac{\mathrm{d}^{2} x_{\mathrm{cyl} D}(t)}{\mathrm{d} t^{2}} \\
\frac{\partial^{i} x_{\mathrm{cyl} R}(t)}{\partial t^{i}}= & \cdots
\end{aligned}
$$

The momentaneous values of the driving cylinder trajectory are available through the actuator trajectory. The evaluation of the gear function leads to $G(u)$ and its derivatives. Thus, to calculate the cylinder trajectory only a few function evaluations and a few multiplications / additions are necessary.

a) Polynomials: For a $C^{2}$ actuator trajectory as in Figure 6, $G(u)$ also has to be $C^{2}$. Additionally, $G(u)$ has to fulfill the earlier presented boundary conditions, in the case of $C^{2}$ there are 3 conditions, and the skew symmetricity conditions. This leads to at least a polynomial of degree 5 being needed, whereby the coefficients $k_{0}, k_{2}$ and $k_{4}$ are zero.

$$
G(u)=\sum_{i=0}^{m} k_{i} u^{i}=k_{1} u+k_{3} u^{3}+k_{5} u^{5}
$$

The polynomial is shown in Figure 11 as dashed curve.

The disadvantage of this polynomial is the high retracting velocity.

b) Piecewise Polynomials - S-Curve: Here the same method as in Section IV-A, Actuator Trajectory, is used, see also [18], [17]. $G(u)$ based on piecewise polynomials of degree 3 is shown in Figure 11. Also in this case, the curve has to fit to the actuator trajectory. For a $C^{2}$ actuator trajectory, a $C^{2}$ curve is therefore necessary. The boundary conditions and skew-symmetricity conditions also have to be fulfilled.

In comparison to the case actuator trajectory, the requirements on the algorithm to generate the curve are low. The generation can be done off-line and the algorithm has only to solve the simple symmetric case similar to "from rest to rest". The problem can be solved by a simple search. The advantage is that the parameters of the S-curve, the limits on its derivatives, correspond to the defined limits for the actuator on the cylinder trajectories, see Equations 14 to 17.

c) Lookup-Tables: are a way to implement arbitrary gear functions with low computational costs on a controller enabling real-time execution. The gear function and its derivatives are stored in tables. A function evaluation is realized by linear interpolation. As the derivatives are stored as separate tables, no numerical derivation is necessary.

\section{TRACKIng CONTROLleR}

The tracking controller has two purposes in this concept. First, tracking of the motion of the cylinders and second, handling the load shift, which implies force control for the cylinders.

Many researchers have published work on control of hydraulic servo systems, see [29], [10], [9], [30]. A common assumption in controllers for hydraulic servo systems is that the valve dynamic is fast compared to the system and can thus be ignored. [31] states: "... the valve's $90^{\circ}$ phase point should exceed the load resonant frequency by a factor of three or more ...". When retracting, the system's natural frequency is around $125 \mathrm{~Hz}$, see [2]. Hence, the valve with a corner frequency for small amplitudes $f_{\mathrm{V}}$ of $200 \mathrm{~Hz}$ does not comply with this rule of thumb. This leads to the selection of the following controller, which works well in that circumstances.

\section{A. The Controller}

The proposed controller is based on the Adaptive Feedback Linearising Control (AFLC) in [30]. Stability, robustness, and performance have been analyzed in [30] and other publications of these authors. For the controller design, a reduced model derived by introducing load pressure $p_{\mathrm{L}}=p_{\mathrm{A}}-p_{\mathrm{B}}$ according [10] is used.

$$
\begin{aligned}
q_{\mathrm{L}} & =C_{\mathrm{V}} x_{\mathrm{V}} \frac{1}{\sqrt{2}} \sqrt{p_{\mathrm{S}}-p_{\mathrm{T}}-\operatorname{sign}\left(x_{\mathrm{V}}\right) p_{\mathrm{L}}} \\
\frac{\mathrm{d}}{\mathrm{d} t} p_{\mathrm{L}} & =\frac{E}{V}\left(2 q_{\mathrm{L}}-2 A_{\mathrm{P}} \frac{\mathrm{d}}{\mathrm{d} t} x_{\mathrm{P}}\right) \\
q_{\mathrm{L}} & =\frac{q_{\mathrm{A}}-q_{\mathrm{B}}}{2}=q_{\mathrm{A}}=-q_{\mathrm{B}}
\end{aligned}
$$

$x_{\mathrm{V}}$ stands for the valve's normalized spool position, and $x_{\mathrm{P}}$ for the piston position and later on, $x_{\mathrm{Pd}}$ of the desired piston position. The pressure dynamics in the cylinder chambers, which are faster than the rest of the system, are ignored by $\frac{\mathrm{d}}{\mathrm{d} t} p_{\mathrm{L}}=0$ and thus Equation 20 combined with Equation 19 results in:

$$
q_{\mathrm{L}}=A_{\mathrm{P}} \frac{\mathrm{d}}{\mathrm{d} t} x_{\mathrm{P}}=C_{\mathrm{V}} x_{\mathrm{V}} \frac{1}{\sqrt{2}} \sqrt{p_{\mathrm{S}}-p_{\mathrm{T}}-\operatorname{sign}\left(x_{\mathrm{V}}\right) p_{\mathrm{L}}}
$$

Substituting $\frac{\mathrm{d}}{\mathrm{d} t} x_{\mathrm{P}}$ with $v$, whereby $v$ is the new input, yields the feedback linearizion law:

$$
x_{\mathrm{V}}=\frac{A_{\mathrm{P}} \sqrt{2}}{C_{\mathrm{V}} \sqrt{p_{\mathrm{S}}-p_{\mathrm{T}}-\operatorname{sign}\left(x_{\mathrm{V}}\right) p_{\mathrm{L}}^{* *}}} v
$$

In Equation 23, $x_{\mathrm{V}}$ appears on both sides of the equation. $A_{\mathrm{P}}$ and $C_{\mathrm{V}}$ are positive constants and for relevant cases the term $\sqrt{\text {. satisfies }} p_{\mathrm{S}}-p_{\mathrm{T}}>0$ and $p_{\mathrm{L}}<p_{\mathrm{S}}-p_{\mathrm{T}} . x_{\mathrm{V}}$ and $v$ therefore have to have equivalent signs and $\operatorname{sign}\left(x_{\mathrm{V}}\right)$ can be replaced with $\operatorname{sign}(v)$.

Applying this feedback law to Equation 22, assuming that the estimated parameters in the feedback law resemble the plant, leads to the new system equation:

$$
\frac{\mathrm{d}}{\mathrm{d} t} x_{\mathrm{P}}=v
$$


The feedback control law has been chosen as:

$$
\begin{aligned}
v & =\frac{\mathrm{d}}{\mathrm{d} t} x_{\mathrm{Pd}}+k_{\mathrm{pos}} e_{\mathrm{pos}}+k_{\mathrm{vel}} e_{\mathrm{vel}}+k_{\mathrm{pre}} e_{\mathrm{pre}} \\
e_{\mathrm{pos}} & =x_{\mathrm{Pd}}-x_{\mathrm{P}}^{*} \\
e_{\mathrm{vel}} & =\frac{\mathrm{d}}{\mathrm{d} t} x_{\mathrm{Pd}}-\frac{\mathrm{d}}{\mathrm{d} t} x_{\mathrm{P}}^{*} \\
e_{\mathrm{pre}} & =p_{\mathrm{Ld}}-p_{\mathrm{L}}^{*}
\end{aligned}
$$

The mechanical system can be modeled as

$$
\begin{aligned}
\frac{\mathrm{d}^{2}}{\mathrm{~d} t^{2}} x_{\mathrm{P}} & =\frac{1}{m}\left(-B \frac{\mathrm{d}}{\mathrm{d} t} x_{\mathrm{P}}+F_{\mathrm{LX}}+F\right) \\
F & =A_{\mathrm{P}} p_{\mathrm{L}}
\end{aligned}
$$

Rearranging this equation and substituting the actual values with the desired values leads to the desired load pressure expressed in terms of the desired trajectory and a known or estimated load $F_{\text {LXe }}$.

$$
p_{\mathrm{Ld}}=\frac{m \frac{\mathrm{d}^{2}}{\mathrm{~d} t^{2}} x_{\mathrm{Pd}}+B \frac{\mathrm{d}}{\mathrm{d} t} x_{\mathrm{Pd}}-F_{\mathrm{LX} \mathrm{e}}}{A_{\mathrm{P}}}
$$

The whole control law is formed by Equations 23, 25 and 27 , whereby the output is the desired valve spool position and the measured variables are the load pressure $p_{\mathrm{L}}^{*}=p_{\mathrm{A}}^{*}-p_{\mathrm{B}}^{*}$, the piston / cylinder position $x_{\mathrm{P}}^{*}$ and velocity $\frac{\mathrm{d}}{\mathrm{d} t} x_{\mathrm{P}}^{*}$.

\section{B. Scheduling}

The system dynamics change, when a cylinder engages or disengages, therefore the controller parameters have to be adjusted accordingly. These changes are in a first approximation not variations in some parameters, like bulk modulus of the fluid, but the system switches within a known set of system dynamics. The changes are triggered by the controller itself, namely by the state transitions of the inverse kinematics. Additionally to the switches in system dynamics, the control objective changes during the cycle.

Figure 3 shows the control scheme for a system with two cylinders. The block scheduler selects the parameter set for each controller depending on the discrete state of the inverse kinematics. In [24] Ioannou presents the applied concept, there called Gain Scheduling. Figure 8 shows the main states and their sequence. In the implementation of the control concept, the stats are subdivided into more specific sub-states. For each discrete state there may, but does not have to, be an independent parameter set.

\section{Adaptation}

Parameters like viscosity, bulk modulus of the fluid, friction and others may vary during operation and thus change the system's behavior. Therefore, the control concept includes parameter adaptation.

The goal in this contribution is to provide smooth or even constant velocity motion. Unfortunately, smooth trajectories tend to contain insufficient excitation for adaptation [32]. This contribution therefore focuses on only one parameter, an external force, $F_{\mathrm{LX} i s}$. Even though only one parameter is considered, this parameter can have different values for each state $s$ and each cylinder $i$.
This external force, $F_{\mathrm{LX} i s}$, is defined as the combination of the load force $F_{\mathrm{L}}$ and a disturbance force $F_{\text {cylis }}^{*}$ acting on the cylinder. This disturbance force aggregates cylinder and guide friction, gravity and so on of one cylinder.

$$
F_{\mathrm{LX} i s}=F_{\text {cylis }}^{*}+e_{i} F_{\mathrm{L}}
$$

The load force $F_{\mathrm{L}}$ is assumed to be quasi-constant through all states and is a single to adapt value. The engaging factor $e_{i}$, generated by the inverse kinematics, governs how strongly the cylinder is engaged in driving the load and is derived from the load distribution.

By making assumptions about the system, the number of values of $F_{\text {cylis }}^{*}$ can be reduced. The assumption made in the simulation model is that the force $F_{\text {cylis }}^{*}$ is quasi-constant for the forward and the backward motion of each cylinder, i.e. there are four distinct cases to consider. The rationale is that there may be gravity acting on the piston and that the friction of the seals may have a direction-dependent part. Thus, there are in total five values to adapt.

It has to be decided when the adaptation takes place. In the simulation model, the state driving is used to adapt $F_{\mathrm{L}}$, the state retracting to adapt $F_{\text {cylis }}^{*}$ for the retracting direction and the state sync, a substate of the state retracting, to adapt $F_{\text {cylis }}^{*}$ for the driving direction.

The adaptation law derived according to [24] is as simple as an integral and resembles the I part of a PID controller. It is implemented as:

$$
F_{\mathrm{LX} i s e, k+1}=F_{\mathrm{LX} i s e, k}+a_{i s}\left(x_{\mathrm{cyl} i d, k}-x_{\mathrm{cyl} i, k}\right) T_{s}
$$

$T_{s}$ stands for sample time, $a_{i s}$ for the adaptation parameters.

\section{Estimator}

For a real-world implementation, an estimator is necessary as it can not be assumed that for example the cylinder velocity can be measured. This contribution builds on simulation and velocity can therefore be measured. For this reason, the estimator is not discussed here.

\section{Simulation AND Results}

The application chosen to analyze and evaluate the proposed control concept is to ascend and then to descend the mass $1 \mathrm{~m}$. The system is modeled ideally but realistically, i.e. the parameter matches existing commercially available off-theshelf components but higher order effects and disturbances are ignored. The pressure sensors are modeled as a second order system with a corner frequency of $1.5 \mathrm{kHz}$, but noise is not modeled. Position and velocity sensors are modeled ideally. The controller is modeled time discrete with a cycle time of $200 \mu \mathrm{s}$, which is realistic as the control algorithm is computationally inexpensive. The controller parameters are selected by simulation-based optimization.

\section{A. Control Performance}

Figure 12 shows simulation results of the analyzed motion. Figures 13 and 14 amplify details. As shown in Figure 14, during the transition phase the load is actively shifted to the 


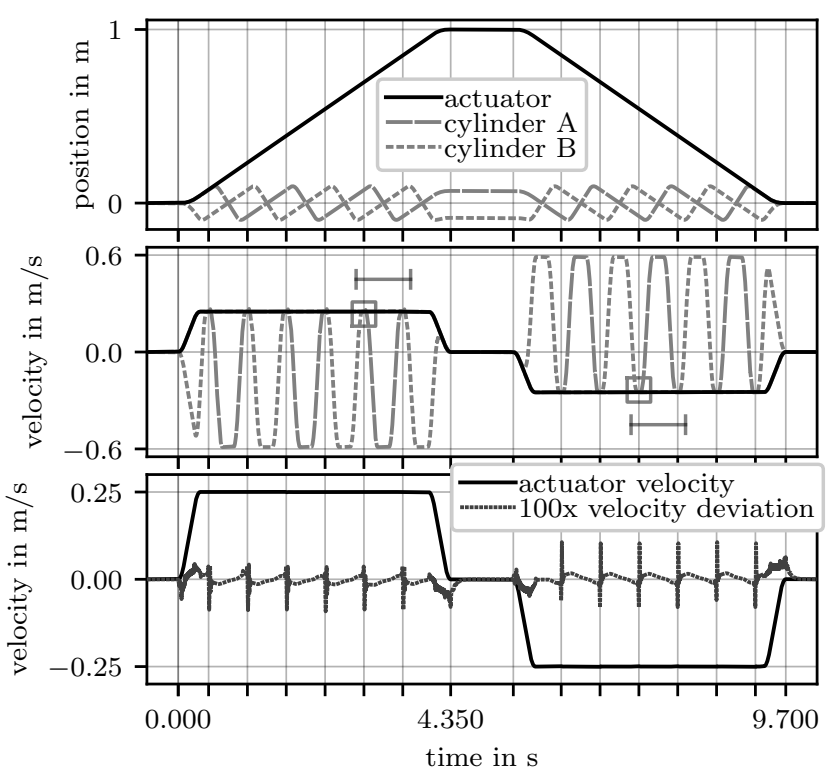

Fig. 12. Simulation result of a motion from position $0 \mathrm{~m}$ to position $1 \mathrm{~m}$ and back. Plot 2 shows the velocity of both cylinders and the actuator. Plot 3 shows the velocity of the actuator and the velocity deviation increased by factor 100. The horizontal bars mark the details shown in Figure 13 and the squares the details in Figure 14.
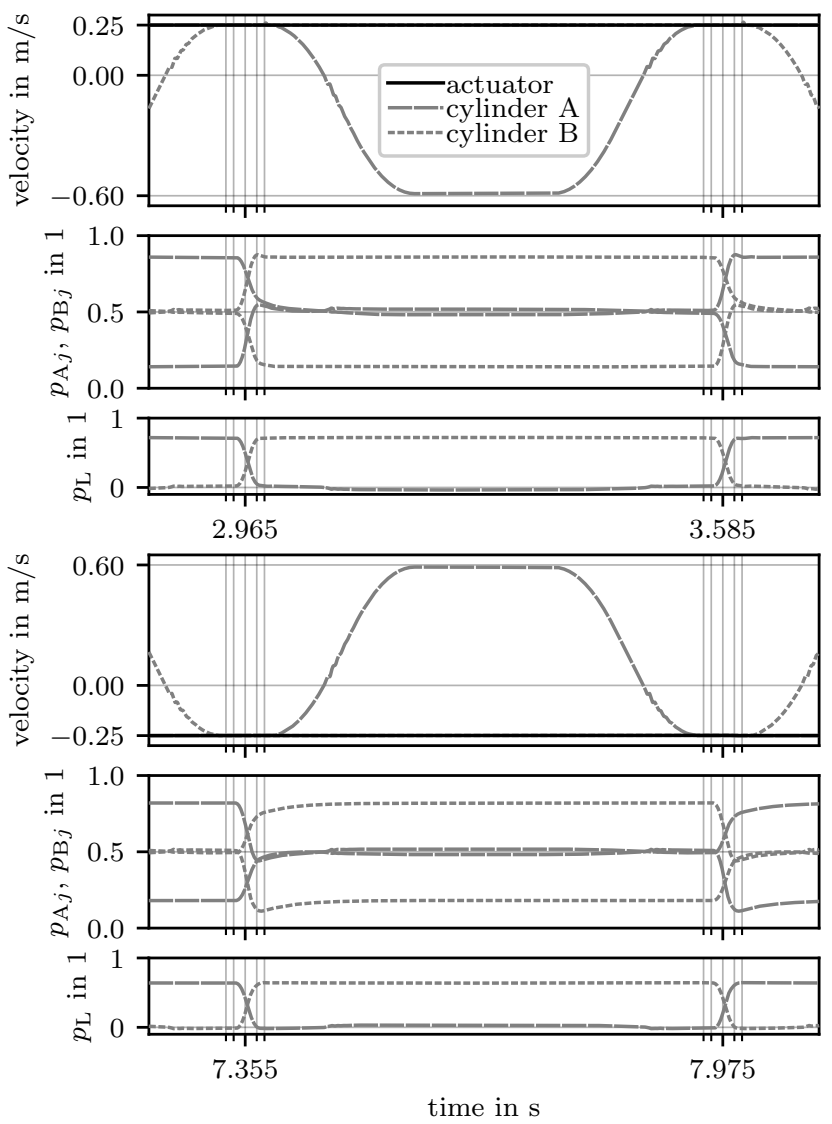

Fig. 13. Detail from Figure 12. Plots 1, 2, and 3 show one cycle of cylinder motion for positive velocity and plots 4,5 , and 6 for negative velocity. Plots 2 and 5 show the chamber pressures $p_{\mathrm{A} j}, p_{\mathrm{B} j}$ in the cylinders and plots 3 and 6 the load pressures normalized to system pressure.
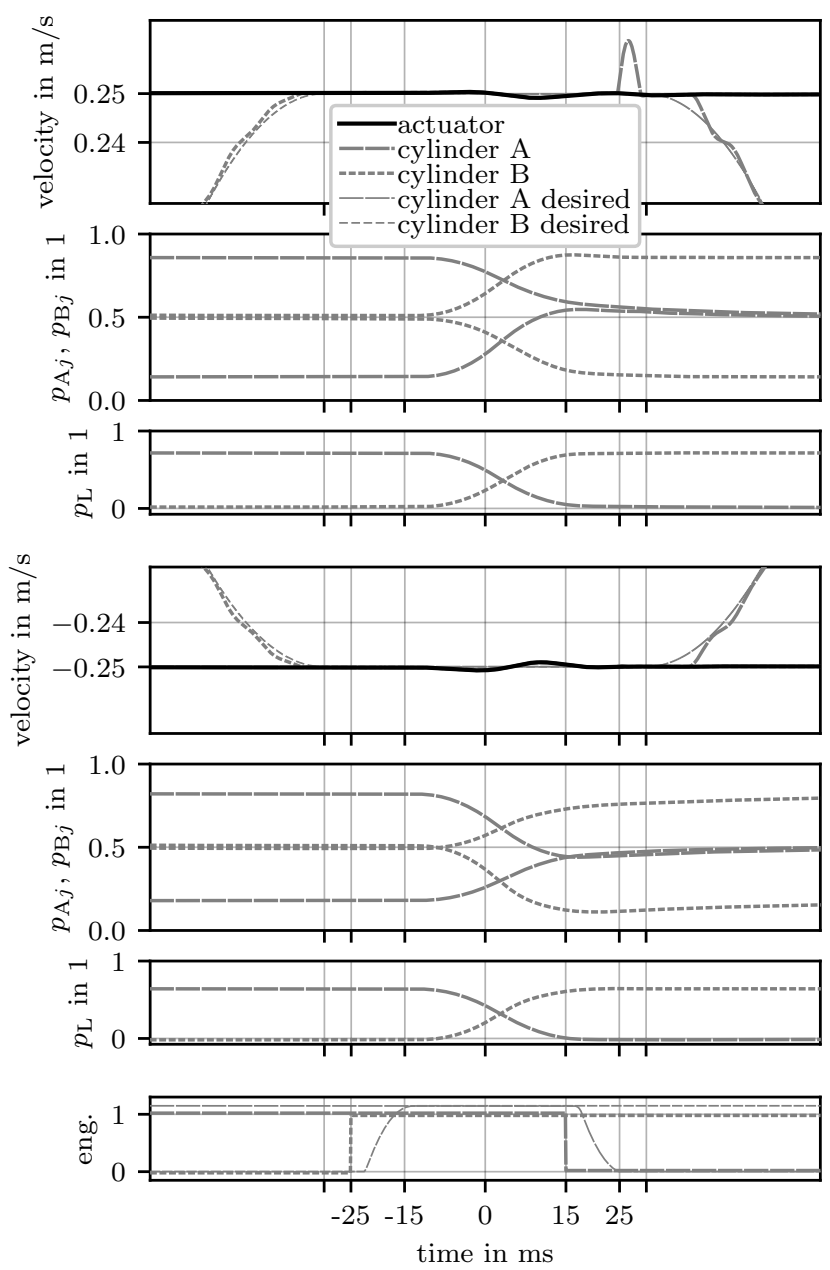

Fig. 14. Details from Figure 12, marked with the squares. Plots 1, 2, and 3 show the situation for positive velocity and plots 4, 5, and 6 for negative velocity. Plot for engagement, 'eng.', is identical for both cases. Chamber pressure $p_{\mathrm{A} j}, p_{\mathrm{B} j}$, plots 2 , and 5 , and load pressure, plots 3 , and 6 , are normalized to system pressure.

future load-driving cylinder. The sum of the cylinder forces nearly matches the load force, but the residual force causes some velocity disturbance in the actuator. Plot 3 in Figure 12 shows this deviation, which remains below approximately $0.001 \mathrm{~m} / \mathrm{s}$. The actuator's position deviation is the sum of slip and the control error. In the proposed control concept, slip is compensated by the actuator trajectory generator in a manner appropriate for the application. The control error caused by velocity variations thus has to be used to evaluate the control performance. Simulation shows that for the case of constant desired velocity after compensating for slip, the perceptible position deviation variation remains within $\pm 15 \mu \mathrm{m}$. These deviations reflect the ideal load model.

Figure 14 shows that in the case of positive velocity, the pressures before and after the moment of disengaging does not perfectly match and thus a small jerk is introduced mainly into the retracting cylinder. The oscillations of the retracting cylinder before synchronizing with the actuator, visible in Figures 14 and 13, are caused by the modeled Coulomb friction in the cylinder. 


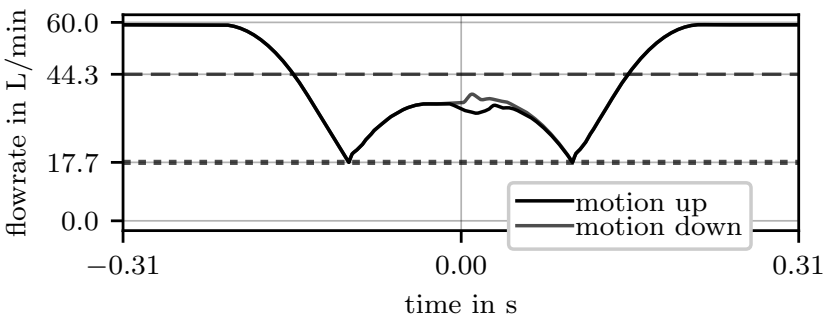

Fig. 15. Flow demand of the actuator. The plot shows the combined flow demand of both cylinders for the case of constant velocity. The dotted line shows the flow demand of the driving cylinder which corresponds to the flow demand of a conventional cylinder. The flow demand beyond that line is used for retraction. The dashed line shows the average flow demand.

\section{B. Unsteady Flow Demand}

Figure 15 shows the flow demand of the actuator for constant velocity. Additionally to the flow for the driving cylinder, the retracting cylinder requires flow, too. For the retracting cylinder the peak velocity is higher and the moving distance is longer than for the driving cylinder. The actuator therefore requires 3.4 times the peak flow and 2.6 times the average flow of the driving cylinder. Flow corresponds to energy usage.

Additionally, the clamping mechanism requires a peak flow of $32 \mathrm{~L} / \mathrm{min}$ for a moment. At an actuator velocity of $0.25 \mathrm{~m} / \mathrm{s}$, this results in a mean flow of $0.13 \mathrm{~L} / \mathrm{min}$.

\section{DISCUSSIONS}

The ambition in this contribution is to realize highperformance motion with HILA, which includes smooth motion. In the sense of a benchmark, the limits of the concept are investigated. In simulation with ideal load, the internal disturbances of the load shift could next to be prevent. Energy efficiency considerations have been intentionally ignored and are a topic for ongoing research.

The proposed hydraulic schema imposes a flow demand of more than twice of a conventional cylinder and results thus in poor energy efficiency. More complex valve systems may reuse the flow of the driving cylinder for retraction.

Two critical parameters are the duration of the transition phase and the additional distance the retracting cylinder has to travel to reverse direction. Both parameters influence the energy efficiency and the feasible actuator velocity. Reducing these parameters leads, for a given effective stroke length, to smaller retraction velocity, and thus smaller peak flow, and to a shorter retraction stroke and thus smaller average flow. This leads to a shorter required cylinder stroke length and thus a smaller actuator. The effective stroke length is the distance the actuator moves the load during one cycle.

Reducing the additional travel distance for reversing requires high-performance servo valves. There is a trade-off between fast reversing and the quality of synchronization from the retracting cylinder with the load, which may introduce disturbances into the system.

The duration of the transition phase is characterized by the duration of the engaging, the disengaging, and the load shift. Fast engaging / disengaging requires fast and relatively big on/off valves and the switching valve FSVi 4.1 [26] from Linz Center of Mechatronics $\mathrm{GmbH}$ is therefore proposed. The duration of the load shift is affected by the performance of the valve system and the controller. Here again, there is a trade-off between fast load shift and low disturbance of the system.

All these parameters, cylinder length, valve and sensor performance, trajectory and controller, are dependent on each other and therefore an integrated design approach is required to design this Mechatronic system.

The concept can be extended to three or more cylinders which lowers the requirements on the retraction trajectory and thus the components and may allow higher performance.

The short cylinder stroke length results in high hydraulic stiffness and thus high natural frequencies. During the phase of retraction, the effective mass is reduced to the mass of only the piston. This leads to natural frequencies similar to the $90 \mathrm{deg}$ phase shift frequencies of common high response servo valves. Active damping is therefore a challenge.

The simulation presents motion of $1 \mathrm{~m}$. As the properties of the actuator do not change with position and stroke length, this represents motion of arbitrary length and position and is characteristic for the actuator.

\section{CONCLUSIONS}

This contribution shows that direct hydraulically actuated infinite linear motion can be realized. It proposes a control algorithm which achieves smooth motion at relatively high velocity. Even position control over long distances is feasible.

The main contribution is the control concept based on inverse kinematics. The requirements on the gear function of the inverse kinematics are derived and two candidates have been presented. The consequences of the choice of gear function have been analyzed. The performance of the proposed controller is analyzed with the help of simulation.

The performance of the actuator is similar to or may even surpass conventional cylinders depending on the application. The application does not have to deal with the additional complexity of the actuator as it is fully encapsulated into the control system.

\section{REFERENCES}

[1] M. Hochwallner and P. Krus, "Motion Control Concepts for the Hydraulic Infinite Linear Actuator," in Proceedings of the 9th FPNI PHD Symposium on Fluid Power, 2016, ISBN: 978-0-7918-5047-3.

[2] M. Hochwallner, M. Landberg, and P. Krus, "The Hydraulic Infinite Linear Actuator - properties relevant for control," in Proceedings of the 10th International Fluid Power Conference (10. IFK), 2016.

[3] M. Hochwallner and P. Krus, "Hydraulic Infinite Linear Actuator The Ballistic Gait - Digital Hydro-Mechanical Motion," in Proceedings of the 15th Scandinavian International Conference on Fluid Power, SICFP'17, 2017.

[4] K. T. Ulrich and S. D. Eppinger, Product Design and Development, 5th ed. McGraw-Hill, 2012, iSBN-13: 978-0071086950.

[5] R. Dove, "Agile Systems-Engineering and Agile-Systems Engineering," INSIGHT - International Council on Systems Engineering (INCOSE), no. Issue 2, 2014.

[6] M. Linjama, H.-P. Vihtanen, A. Sipola, and M. Vilenius, "Secondary Controlled Multi-Chamber Hydraulic Cylinder," in The 11th Scandinavian International Conference on Fluid Power, SICFP09, Linköping, Sweden, 2009.

[7] H. Kogler, "The Hydraulic Buck Converter - Conceptual Study and Experiments," Ph.D. dissertation, 2012, ISBN: 978-3990330593. 
[8] B. Eriksson and J.-O. Palmberg, "Individual Metering Fluid Power Systems: Challenges and Opportunities," Proceedings of the Institution of Mechanical Engineers. Part I, Journal of Systems and Control Engineering, vol. 225, no. 12, pp. 196-211, 2011.

[9] A. Kugi, Non-linear Control Based on Physical Models: Electrical, Mechanical and Hydraulic Systems, ser. Lecture Notes in Control and Information Sciences. Springer, 2000, no. 260, ISBN: 99-0147115-X

[10] M. Jelali and A. Kroll, Hydraulic Servo-systems: Modelling, Identification and Control, ser. Advances in Industrial Control. Springer, 2004 ISBN: 978-1-4471-1123-8.

[11] C. Gradl, A. Plöckinger, and R. Scheidl, "Sensorless position control with a hydraulic stepper drive - Concept, compression modeling and experimental investigation," Mechatronics, vol. 35, pp. 91 - 101, 2016.

[12] T. Pfeifer and R. Schmitt, Autonome Produktionszellen. Springer-Verlag GmbH, 2005, ISBN-13: 978-3540292142.

[13] J. Holton and J. Inman, "Piston-Cylinder Assembly," Patent US 4526086 A, 1985, US Patent 4,526,086.

[14] G. Reymann and P. Baumeister, "Hydraulischer Linearmotor," Patent, 1978, german Patent DE 2649958 A1.

[15] Website of ENERPAC - Strand Jacks. http://www.enerpac.com/en/integrated-solutions/strand-jacks.

[16] T. Kröger and F. M. Wahl, "Online Trajectory Generation: Basic Concepts for Instantaneous Reactions to Unforeseen Events," IEEE Transactions on Robotics, vol. 26, no. 1, pp. 94-111, Feb. 2010.

[17] B. Ezair, T. Tassa, and Z. Shiller, "Planning High Order Trajectories with General Initial and Final Conditions and Asymmetric Bounds," The International Journal of Robotics Research, vol. 33, no. 6, 2014.

[18] K. D. Nguyen, I.-M. Chen, and T.-C. Ng, "Planning Algorithms for S-curve Trajectories," in 2007 IEEE/ASME international conference on advanced intelligent mechatronics. Institute of Electrical \& Electronics Engineers (IEEE), 2007.

[19] D. Liberzon, Switching in Systems and Control, ser. Systems \& Control: Foundations \& Applications. Birkhäuser Boston, Inc., Boston, MA, 2003, iSBN: 0-8176-4297-8.

[20] "Website of NoMagic - Cameo Systems Modeler," http://www.nomagic.com/products/cameo-systems-modeler.html.

[21] S. Friedenthal, A. Moore, and R. Steiner, A Practical Guide to SysML: The Systems Modeling Language, 2nd ed. Morgan Kaufmann, OMG, 2011, ISBN: 978-0123852069.

[22] R. Stadlmayr, "On a Combination of Feedforward and Feedback Control for Mechatronic Systems," Ph.D. dissertation, 2008, ISBN-13: 9783832280659

[23] K. J. Åström and R. M. Murray, Feedback Systems : An Introduction for Scientists and Engineers. Princeton University Press, 2008, ISBN: 9780691135762

[24] P. A. Ioannou and J. Sun, Robust Adaptive Control, ser. Dover Books on Electrical Engineering Series. Dover Publications, 2012, ISBN-13: 978-0486498171.

[25] ETP Transmission AB, "ETP-OCTOPUS - Datasheet for Octopus."

[26] Linz Center of Mechatronics GmbH - Hydraulic Drives, "FSVi 4.1 Datasheet - Fast Switching Valve Technolog," 2016, http://www.lcm.at/.

[27] B. Winkler, A. Plöckinger, and R. Scheidl, "State Of The Art In Digital Valve Technology," in PROCEEDINGS - The Seventh Workshop on Digital Fluid Power, 2015, pp. 151-163.

[28] C. de Boor, Splinefunktionen, ser. Lectures in mathematics. Basel; Boston: Birkhäuser, 1990, 1990, ISBN: 3764325143.

[29] H. E. Merritt, Hydraulic Control Systems, 1967, ISBN: 0471596175.

[30] T. O. Andersen, M. R. Hansen, H. C. Pedersen, and F. Conrad, "On the Control of Hydraulic Servo Systems: Evaluation of Linear and Nonlinear Control Schemes," in Proceedings of The Ninth Scandinavian International Conference on Fluid Power, SICFP05, 2005.

[31] MOOG, "Electrohydraulic Valves: A Technical Look," 2013, http://www.moog.com/literature/ICD/Valves-Introduction.pdf.

[32] S. Mayr and G. Grabmair, "Trajectory generation with rich information content," PAMM, vol. 14, no. 1, pp. 917-918, dec 2014.

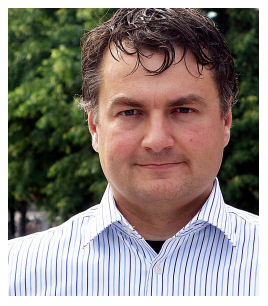

Martin Hochwallner received his Dipl.-Ing. degree in Mechatronics from Johannes Kepler University in Linz, Austria, in 2004. He is currently working toward the Ph.D. degree at the Division of Fluid and Mechatronic Systems, Linköping University (LiU), Sweden. He worked in industry in product development of heavy industrial machinery and was with the Linz Center Of Mechatronics $\mathrm{GmbH}$, Austria. His research activities are focused on mechatronics, fluid power, control, and system engineering.

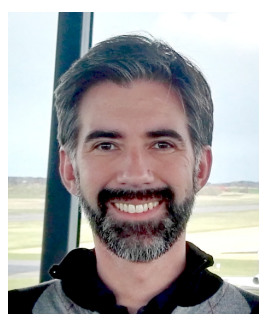

Lie Pablo Grala Pinto received M.Sc degree from Peter the Great St.Petersburg Polytechnic University (SPbPU), Russia in 2008 and D.Eng. at Federal University of Santa Catarina (UFSC), Brazil in 2015 all in automatic control. He is currently a Postdoctoral research at the Division Fluid and Mechatronic Systems (Flumes) at Linköping University (LiU), Sweden. His research interests include switched systems, robust control, system stability, sliding modes control, system identification and state observers.

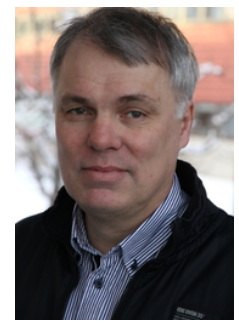

Petter Krus is chaired professor in Fluid and Mechatronic Systems at Linköping University, since 2010. He has a PhD in Hydraulic Control Systems in 1988 from Linköping University, and became full professor in Machine Design in 2001. Since 2015 he is also Swedish Professor of Aeronautics - Endowed Chair in Memory of Peter Wallenberg Sr at Instituto Tecnológico de Aeronáutica in Brazil. Research interest in fluid power, mechanical, and mechatronic systems technology, specifically focusing on system dynamics, control, system simulation, optimization, system design and design automation (system and geometric (CAD) design) 